\title{
Simulation numérique des ancres à succion en deux- et trois- dimensions en utilisant la méthode des éléments finis
}

\author{
Pierre FORAY, Luisa N. EQUIHUA ANGUIANO \& Marc BOULON \\ Laboratoire 3S-R, (Sols, Solides, Structures - Risques), UMR 5521 \\ Domaine Universitaire B.P. No. 53 \\ 38041 Grenoble cedex 9, France \\ Pierre.Foray@geo.hmg.fr
}

\section{Résumé :}

Les ancres à succion sont des ancrages utilisés pour des systèmes flottants dans les grands fonds marins. Cet article présente une étude paramétrique en conditions $2 \mathrm{D}$ et $3 \mathrm{D}$ des ancres à succion en utilisant le logiciel Plaxis ${ }^{\circledR}$. La résistance non drainée du sol est considérée comme variant linéairement avec la profondeur. Les modèles constitutifs utilisés sont les modèles de comportement de "MohrCoulomb" et celui de "Soft-Soil" proposés par le logiciel. Nous présentons des facteurs de charge qui permettent de prévoir les capacités ultimes sous une charge inclinée, valables en 3D à partir de simulations 2D, en prenant en compte l'apport de la succion sur la capacité. Finalement des enveloppes de rupture sont présentées et comparées avec des solutions théoriques.

\begin{abstract}
:
Suction caissons are structures used as anchoring for floating systems in deepwater petroleum exploitation. In this article, a numerical study of suction caissons is presented. 2D and 3D simulations were performed with the program Plaxis ${ }^{\circledR}$. The soil characteristics considered were clays with a shear strength increasing with depth. The clay was simulated with the two constitutive soil models "Mohr-Coulomb" and "Soft-Soil". Relationships were obtained that permitted the evaluation of the bearing capacity of laterally loaded caissons. 3D results are acquired by extrapolating $2 \mathrm{D}$ simulation. The effect of suction in the bearing capacity is considered. The typical yield failure envelopes are also presented.
\end{abstract}

\section{Mots-clés :}

Ancres à succion - Grands fonds marins - Argiles molles - Contraintes effectives - Simulation numérique - Eléments finis. 


\section{$1 \quad$ Introduction}

Le développement des prospections pétrolières en haute mer a évolué au cours de la dernière décade vers de nouvelles profondeurs d'exploitation, au-delà de 1500 m d'eau (THOMAS, 2007). La particularité des sédiments marins rencontrés à de telles profondeurs est leur faible résistance mécanique, leur très forte plasticité et leur sensibilité au remaniement (COLLIAT \& DENDANI, 2002). Dans ce contexte, l'ancrage des plates-formes flottantes au moyen d'ancres à succion (Fig.1) représente une solution intéressante compte tenu de leur relative facilité d'installation et de leur capacité à l'arrachement.

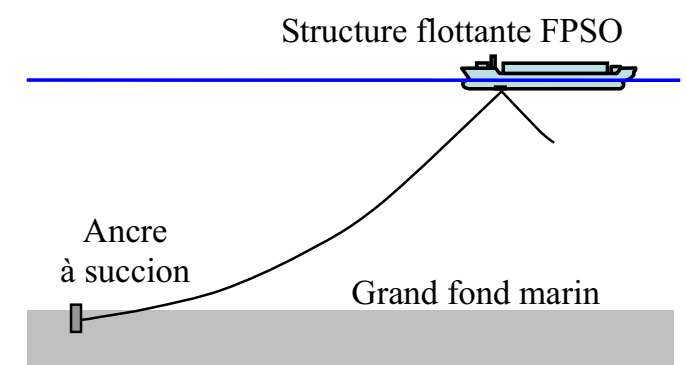

Figure 1. Principe d'utilisation des ancres à succion dans les grands fonds marins

Nous présentons ici une modélisation numérique du comportement des ancres à succion installées dans les argiles molles grands fonds en utilisant les codes de calcul Plaxis ${ }^{\circledR} 2 \mathrm{D}$ et $3 \mathrm{D}$. Les calculs avaient deux objectifs spécifiques : d'une part établir des relations permettant d'évaluer la capacité ultime en conditions réelles $3 \mathrm{D}$, à partir d'une simulation plus rapide faite en conditions $2 \mathrm{D}$, et d'autre part, d'établir numériquement des enveloppes de rupture spécifiques à ces ancres pour des chargements mixtes horizontaux et verticaux. Pour le premier point, on présente une étude paramétrique du facteur de charge, rapport des capacités $3 \mathrm{D}$ et 2D. Cela, au niveau pratique, procure l'avantage d'économiser du temps de calcul, en prenant en compte les conditions réelles $3 \mathrm{D}$ du comportement des ancrages. Pour le second point, les enveloppes de rupture calculées sont comparées avec des solutions théoriques et sont d'une grande utilité dans la conception finale des ancres à succion selon l'inclinaison de la charge appliquée.

Les résultats obtenus pourront être appliqués dans d'autres cadres d'utilisation des ancres. En effet leur principe de fonctionnement peut être extrapolé à des profondeurs d'eau plus faibles. Des projets sont en cours pour appliquer ce type de solutions à des fondations de structures côtières pour les sources d'énergies nouvelles, comme les éoliennes ou les hydroliennes.

\section{Ancres à succion}

Les ancres à succion (Fig.2) utilisées dans les grands fonds marins sont 
typiquement des gros cylindres en acier dont le fond est ouvert et la partie supérieure peut être fermée ou ouverte pour permettre l'application de la succion favorisant leur installation (AUBENY et al., 2003). Leur diamètre important et leur élancement faible par rapport à une fondation profonde classique offrent l'avantage de pouvoir mobiliser de fortes capacités à l'arrachement pour des profondeurs d'ancrages relativement faibles, en combinant une résistance latérale de butée, le frottement le long des parois et éventuellement une succion passive.

Les mécanismes de rupture dépendent de la charge appliquée : verticale (Fig.3) ou inclinée (Fig.4) et des conditions de drainage considérées dans le sol. Le mode de rupture retenu est le plus défavorable. La modélisation en éléments finis permet de retrouver ces modes de rupture et d'évaluer les déplacements de l'ancre (EQUIHUA ANGUIANO \& FORAY, 2007a, 2007b ; EQUIHUA ANGUIANO, 2008).
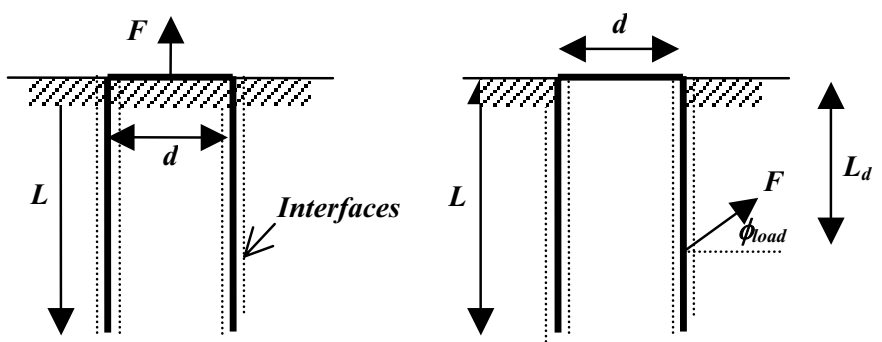

Figure 2. Ancre à succion avec une charge verticale et inclinée $F$

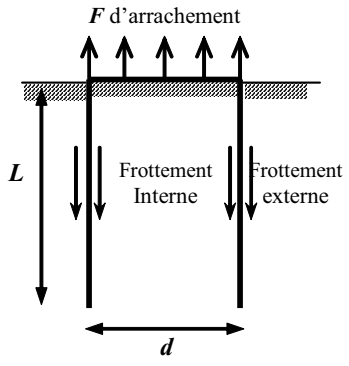

(a) Condition drainée

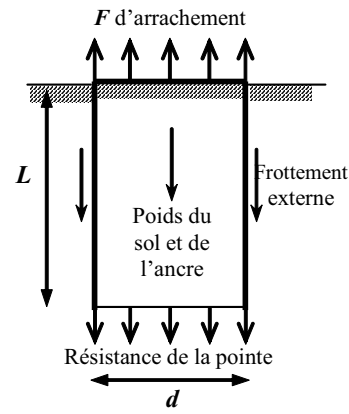

(b) Condition partiellement drainée

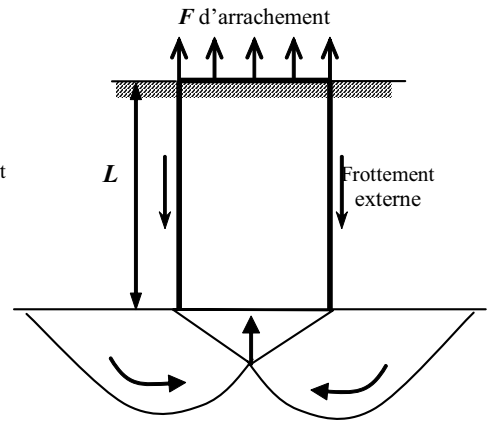

(c) Condition non drainée

Figure 3. Modes de rupture considérés pour une charge verticale à l'arrachement

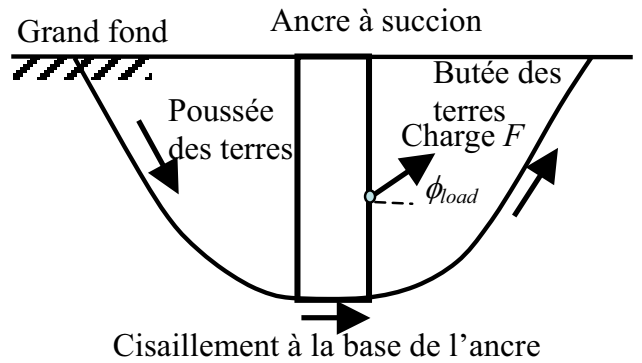

Figure 4. Mode de rupture considéré pour une charge inclinée à l'arrachement 


\section{$3 \quad$ Paramètres utilisés dans les simulations en éléments finis}

Les paramètres utilisés dans les simulations par éléments finis sont présentés dans les paragraphes suivants. Deux modèles constitutifs ont été utilisés : le modèle élasto-plastique parfait avec un critère de Mohr-Coulomb noté par la suite en abrégé "MC" et le modèle "Soft-Soil" de même référencé "SSM", dérivé du modèle Cam Clay et mieux adapté à la simulation du comportement des argiles molles des grands fonds marins. Les paramètres effectifs correspondant à ces deux modèles ont été déterminés de façon à reproduire les profils pénétrométriques donnant une croissance linéaire de la cohésion non drainée avec la profondeur, $c_{u}$ $=1.6 z$, telle que celle observée lors de l'étude du champ pétrolifère Girassol (COLLIAT \& DENDANI, 2002).

\subsection{Paramètres du modèle élasto-plastique et critère de "Mohr-Coulomb"}

La donnée essentielle de départ est le profil de variation de la résistance au cisaillement non drainée avec la profondeur $c_{u}=1.6 z$. Le module d'Young nondrainé du sol a été corrélé à la cohésion non drainée par la relation $E_{u}=215 c_{u}$, en accord avec des résultats d'essais de laboratoire, ce qui a permis ensuite de proposer une expression de la variation du module effectif sécant $E_{50}{ }_{50}$ avec la profondeur $z$ donnée par l'équation (1).

$$
E_{50}^{\prime}=776.6 z+506.7
$$

Une cohésion effective c' non nulle a été introduite pour tenir compte de la légère surconsolidation du sol en surface. Les paramètres utilisés sont indiqués dans le Tableau 1.

Tableau 1. Paramètres géotechniques effectifs, modèle élasto-plastique "MC"

\begin{tabular}{|c|c|c|c|c|c|}
\hline$\gamma_{\text {sat }}\left(\mathrm{kN} / \mathrm{m}^{3}\right)$ & $\boldsymbol{k}_{\boldsymbol{x}}, \boldsymbol{k}_{\boldsymbol{y}}(\mathrm{m} /$ jour $)$ & $\boldsymbol{\phi}^{\prime}\left({ }^{\circ}\right)$ & $\boldsymbol{c}^{\boldsymbol{}}\left(\mathrm{kN} / \mathrm{m}^{2}\right)$ & $\boldsymbol{E}^{{ }_{50}}\left(\mathrm{kN} / \mathrm{m}^{2}\right)$ & $\boldsymbol{v}^{\boldsymbol{\prime}}(-)$ \\
\hline 17 & $8.64^{-5}$ & 27 & 1.6 & $E q .(1)$ & 0.35 \\
\hline
\end{tabular}

\subsection{Paramètres effectifs du modèle "Soft-Soil"}

Le modèle "SSM" requiert, en plus de la cohésion et de l'angle de frottement effectifs $c$ ' et $\phi^{\prime}$ du sol, les paramètres suivants : l'indice de compression modifié "SSM" $\lambda^{*}=\lambda /(1+e)$ et l'indice de gonflement modifié "SSM" $\kappa^{*}=\kappa /(1+e), \lambda$ et $\kappa$ étant les indices de compression et de gonflement du modèle Cam-Clay et e l'indice des vides de l'argile. $\lambda$ a été obtenu à partir de l'indice de plasticité $I_{p}$ en utilisant la corrélation $\lambda=0.6 I_{p}$ et nous avons obtenu $\kappa^{*}$ en adoptant la valeur de 3 pour le rapport $\lambda * / \kappa^{*}$. L'ensemble des paramètres pris pour le modèle "SSM" est indiqué dans le Tableau 2. 
Tableau 2. Paramètres géotechniques effectifs, modèle "Soft-Soil"

\begin{tabular}{|c|c|c|c|c|c|c|c|c|}
\hline$\gamma_{\text {sat }}\left(k N / m^{3}\right)$ & $\boldsymbol{e}(-)$ & $\boldsymbol{I}_{\boldsymbol{p}}(\%)$ & $\boldsymbol{k}_{\boldsymbol{x}}, \boldsymbol{k}_{\boldsymbol{y}}(\mathrm{m} / \mathrm{jour})$ & $\boldsymbol{\phi}^{\prime}\left({ }^{\circ}\right)$ & $\boldsymbol{c}^{\prime}\left(\mathrm{kN} / \mathrm{m}^{2}\right)$ & $\lambda^{*}(-)$ & $\boldsymbol{\kappa}^{*}(-)$ & $\lambda^{*} / \boldsymbol{\kappa}^{*}(-)$ \\
\hline 17 & 3.09 & 96 & 0 & 27 & 3 & 0.141 & 0.047 & 3 \\
\hline
\end{tabular}

\subsection{Géométrie des ancres}

La géométrie et l'élancement $L / d$ des ancres utilisées sont données dans le Tableau 3.

Tableau 3. Elancements et géométries des ancres à succion

\begin{tabular}{|c|c|c|c|c|}
\hline $\boldsymbol{L} / \boldsymbol{d}$ & $\mathbf{2 . 0}$ & $\mathbf{2 . 5}$ & $\mathbf{2 . 7}$ & $\mathbf{3 . 0}$ \\
\hline $\boldsymbol{L}(\boldsymbol{m})$ & 16 & 20 & 16 & 18 \\
\hline $\boldsymbol{d}(\boldsymbol{m})$ & 8 & 8 & 6 & 6 \\
\hline
\end{tabular}

Les ancres ont été modélisées par des éléments "poutre". La modélisation des raidisseurs internes n'a pas été réalisée, mais leur effet a été pris en compte en introduisant une rigidité en flexion EI très grande dans les éléments " poutre" de l'ancre pour éviter leur flambement. Les paramètres caractéristiques de l'acier sont présentés dans le Tableau 4 ( $t$ est l'épaisseur de l'acier).

Tableau 4. Paramètres du matériau utilisé pour les ancres à succion

\begin{tabular}{|c|c|c|c|c|}
\hline $\boldsymbol{w}_{\boldsymbol{a}}\left(\mathrm{kN} / \mathrm{m}^{3}\right)$ & $\boldsymbol{t}(\mathrm{m})$ & $\boldsymbol{v}_{\boldsymbol{a}}(-)$ & $\boldsymbol{G}_{\boldsymbol{a}}\left(\mathrm{kN} / \mathrm{m}^{2}\right)$ & $\boldsymbol{E}_{\boldsymbol{a}}\left(\mathrm{kN} / \mathrm{m}^{2}\right)$ \\
\hline 68.5 & 0.03 & 0.3 & $8.269 \times 10^{10}$ & $2.15 \times 10^{11}$ \\
\hline
\end{tabular}

L'interaction sol-structure au niveau de l'interface sol-acier a été prise en compte par l'introduction du paramètre facteur d'adhérence $\alpha$ (paramètre $\mathrm{R}_{\text {inter }}$ dans Plaxis ${ }^{\circledR}$ ) entre l'argile et l'acier. Les calculs présentés ont été effectués avec deux valeurs du facteur d'adhérence de $\alpha=1.0$ (adhérence parfaite) et 0.7 (adhérence couramment adoptée en pratique).

\section{$4 \quad$ Simulations numériques réalisées}

On résume ci-après une séquence des simulations et études paramétriques effectuées en éléments finis, pour obtenir les facteurs de charge dénommés par la suite "FCU", permettant de passer d'un calcul simplifié en $2 \mathrm{D}$ aux résultats d'un calcul 3D, ainsi que la proposition d'une courbe enveloppe de rupture.

\subsection{Etude paramétrique des charges verticales : axisymétriques et 3D}

Le premier travail a consisté à optimiser le maillage en éléments finis en vérifiant que, pour une charge d'arrachement axiale verticale, les résultats obtenus 
à l'aide de calculs $3 \mathrm{D}$ et $2 \mathrm{D}$ axisymétriques étaient similaires aux résultats $d u$ calcul 3D. Différentes densités et géométries des maillages en éléments finis ont été étudiés.

\subsection{Etude paramétrique des charges inclinées : 2D et 3D}

A partir des maillages et modèles éléments finis obtenus en 4.1, nous avons effectué des calculs 2D (déformation plane dans le plan d'application de la charge) et 3D pour différentes géométries et inclinaisons de charge. Les courbes Charge-Déplacement obtenues ont été ajustées en utilisant l'expression hyperbolique (KONDER, 1963) représentée par l'équation (2) :

$$
F=\delta /(a+b \delta)
$$

où :

$F$ est la charge appliquée au point d'ancrage,

$\delta$, le déplacement du point d'ancrage dans la direction du chargement, $a$, l'inverse de la pente initiale de la courbe Charge-Déplacement, $1 / b$, la capacité ultime pour un grand déplacement.

Les ancres ont été simulées avec une charge $F$ inclinée, un angle d'inclinaison $\phi_{\text {load }}=40^{\circ}$, à une profondeur d'attache $L_{d}=2 / 3 L$ (voir Fig. 1 ).

\subsection{Obtention du facteur de charge "FCU"}

La comparaisons des simulations $2 \mathrm{D}$ et 3D pour les deux modèles constitutifs ("MC" et "SSM") ont permis de calculer les facteurs de charge "FCU", à partir de l'équation (3).

$$
F C U=F_{3 D} / F_{2 D}
$$

où :

$F_{3 D}$ est la charge ultime à l'arrachement en conditions tridimensionnelles,

$F_{2 D}$, la charge ultime à l'arrachement en conditions bidimensionnelles.

\section{$5 \quad$ Résultats}

La Figure 5 montre des exemples de modèles aux éléments finis générés dans Plaxis ${ }^{\circledR} 3 \mathrm{D}$ pour un élancement $L / d=2.0$ et pour une charge $F$ verticale à l'arrachement.

Les Figures 6 montrent les courbes Charge-Déplacement obtenues pour une charge verticale en $2 \mathrm{D}$ axisymétrique et en $3 \mathrm{D}$ avec (a) l'élancement $L / d=2.7$ avec un facteur d'adhérence $\alpha=1.0$ et (b) la représentation normalisée par la force maximale et le déplacement maximum. On vérifie ainsi que les simulations tridimensionnelles (3D) et axisymétriques présentent une bonne correspondance 
au niveau des courbes Charge-Déplacement.
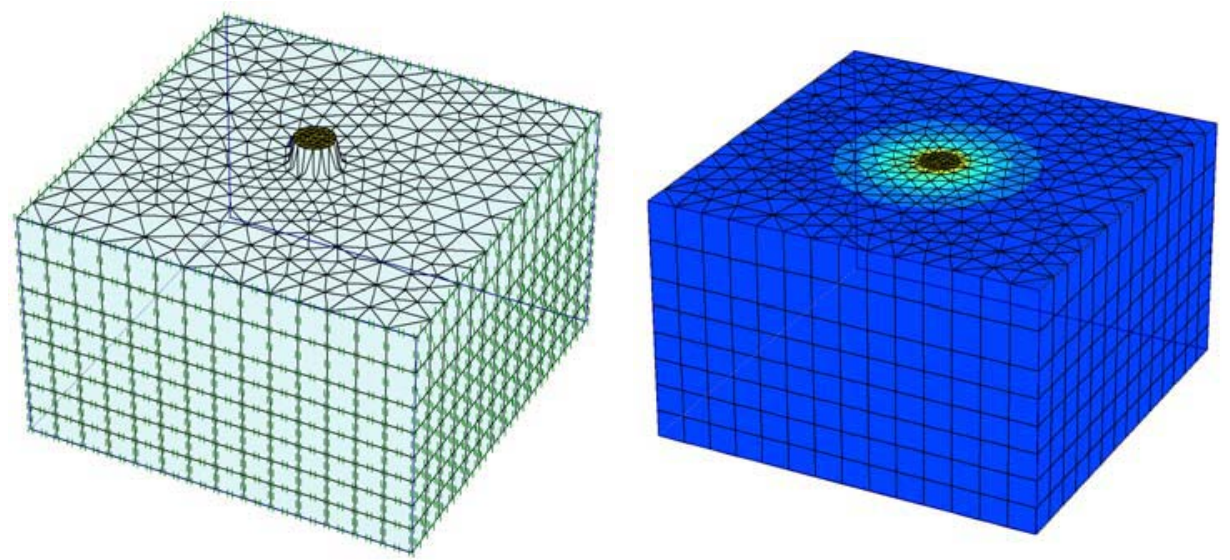

Total incremental displacements (dUtot) Exirerre culot $10.69^{*} 10^{-3} \mathrm{~m}$

Figure 5. Elancement d'ancre $L / d=2.0$, charge verticale d'arrachement, 5840 éléments, 16805 næuds

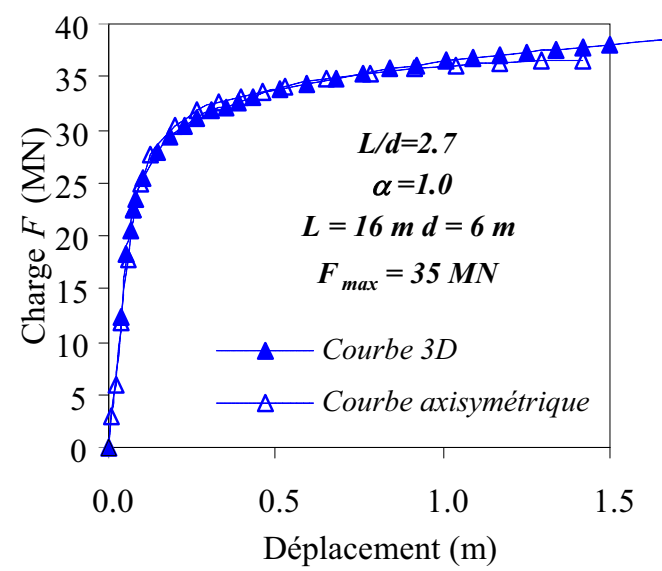

(a)

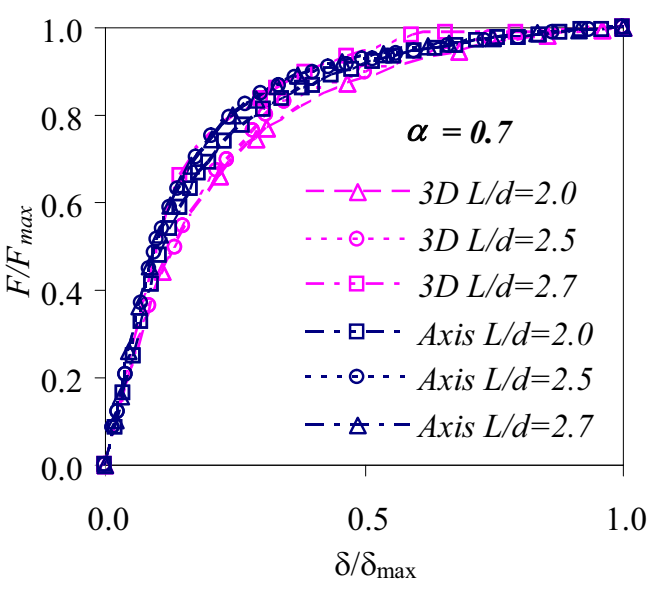

(b)

Figure 6. Courbes Charge-Déplacement et courbes normalisées par rapport à la charge maximale $F_{\max }$, conditions $3 D$ et axisymétriques. Arrachement vertical

Pour simuler les charges inclinées, on a trouvé qu'il était nécessaire d'utiliser une géométrie de maillage avec une frontière horizontale de 10 fois le diamètre $d$ de l'ancre à succion et une frontière verticale de $50 \mathrm{~m}$.

La Figure 7 montre les courbes Charge-Déplacement ajustées obtenues à partir de Plaxis ${ }^{\circledR} 3 \mathrm{D}$ et $2 \mathrm{D}$, pour une charge inclinée $F$, une ancre d'élancement $L / d=$ 2.0 et un facteur d'adhérence $\alpha=1.0$. La notation est la suivante : "2D SSM" pour une simulation bidimensionnelle (2D), avec le modèle constitutif "SSM". 
La Figure 8 présente les variations du Facteur de Capacité Ultime à l'arrachement latéral (FCU) en fonction du déplacement, entre les simulations en condition tridimensionnelle (3D) et en déformation plane (2D), pour les différents modèles constitutifs de sol et pour les élancements $L / d=2.0,2.5,2.7$ et 3.0. Les facteurs d'adhérence dans cette figure sont $\alpha=1.0$ et 0.7 .

La variation de "FCU” peut être résumée par les relations suivantes :

$$
\begin{aligned}
& F C U=F_{3 D-M C} / F_{2 D-S S M}=1.96 \\
& F C U=F_{2 D-M C} / F_{2 D-S S M}=1.52 \\
& F C U=F_{3 D-M C} / F_{2 D-M C}=1.29
\end{aligned}
$$

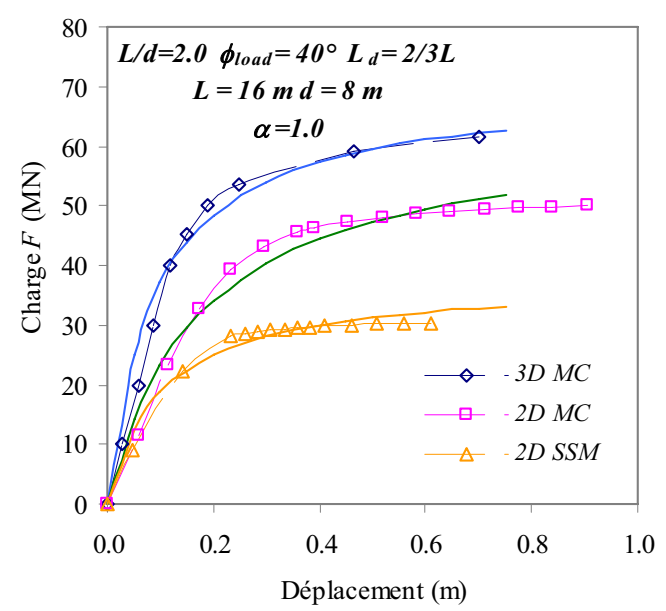

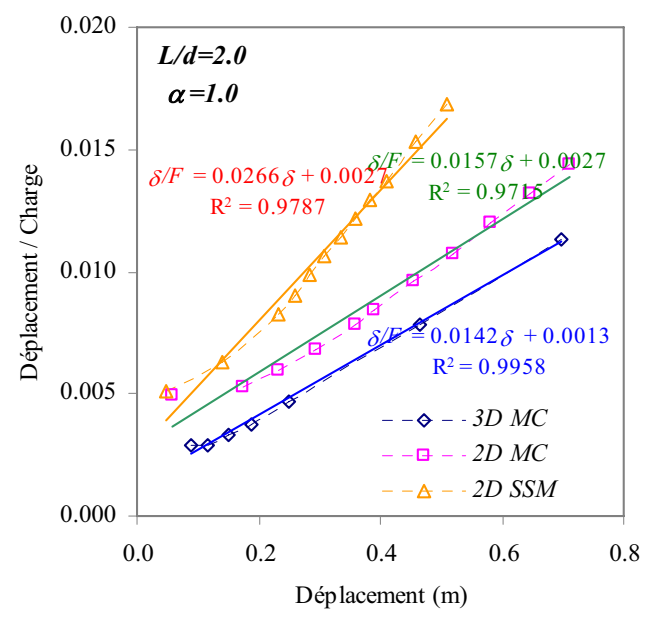

Figure 7. Ajustement des courbes Charge-Déplacement, $3 D$ et $2 D$

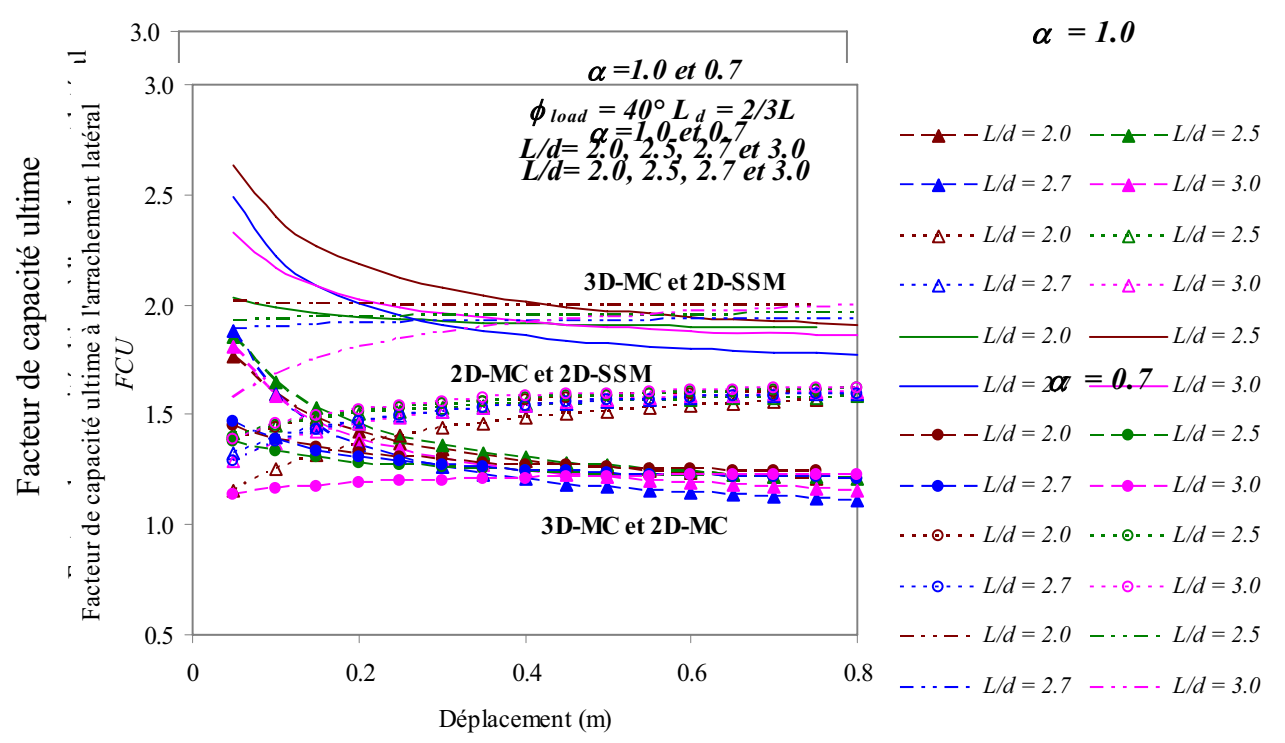

Figure 8. Variation de FCU en fonction du déplacement 


\section{$6 \quad$ Enveloppes de rupture}

Finalement nous présentons des enveloppes de rupture qui permettent d'évaluer la capacité optimale des ancres selon l'inclinaison des charges appliquées. Nous avons obtenu ces enveloppes à partir de simulations en conditions 3D avec Plaxis $^{\circledR}$ en faisant varier l'angle d'inclinaison de la charge $F$, et les avons comparées avec celles obtenues à partir d'expressions analytiques (SUPACHAWAROTE et al., 2004).

$\left(\frac{H}{H_{u l t}}\right)^{a}+\left(\frac{V}{V_{u l t}}\right)^{b}=1$

Les coefficients utilisés sont (SUPACHAWAROTE et al., 2004) :

$a=0.5+L / d$

$b=4.5-L / 3 d$

La Figure 9 présente les enveloppes de rupture $(a)$ sous forme brute et $(b)$ sous forme normalisée par rapport aux efforts verticaux et horizontaux ultimes, obtenues par les simulations 3D pour l'élancement $L / d=5.14, L=21.1 \mathrm{~m}$ et $d=$ $4.1 \mathrm{~m}$.

Une assez bonne concordance entre les enveloppes de rupture obtenues à partir de l'expression analytique et celles obtenues des simulations numériques 3D est observée.
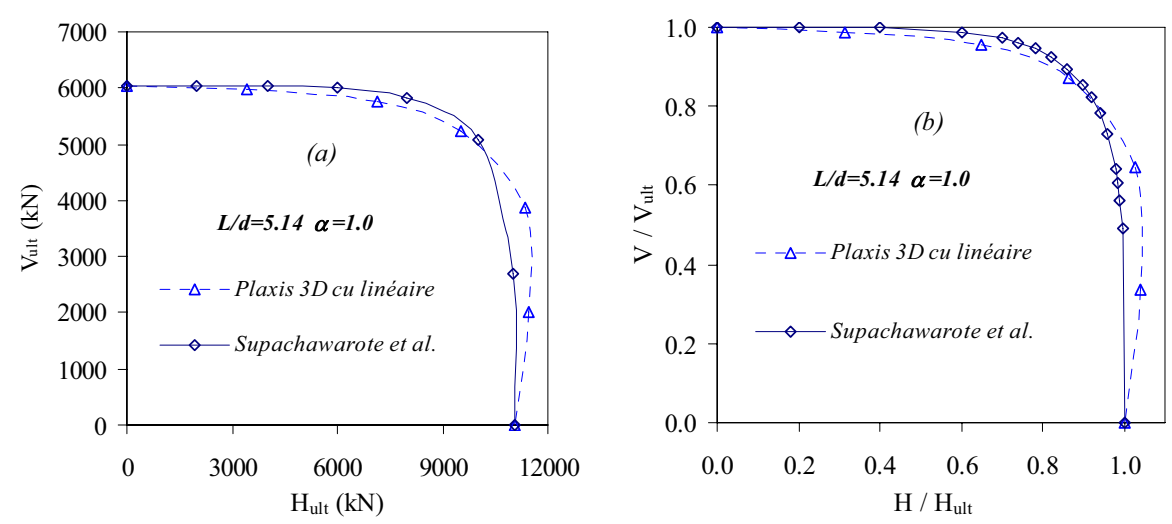

Figure 9. Enveloppes de rupture $L / d=5.14$ avec le modèle "Mohr-Coulomb"

\section{$7 \quad$ Conclusions}

Nous pouvons conclure au sujet de la simulation des ancres avec une charge verticale à l'arrachement, que : les conditions axisymétriques donnent de bonnes correspondances avec les courbes obtenues dans les simulations en conditions 3D. Cette bonne correspondance dépend des caractéristiques des modèles aux éléments finis choisis dans les simulations.

Pour des charges inclinées, à partir des simulations numériques et des 
comparaisons 3D et 2D, nous avons défini et obtenu des Facteurs de Capacité Ultime à l'arrachement latéral (FCU) pour des simulations avec des paramètres effectifs de sol.

Les facteurs FCU permettent, à partir des résultats de capacité ultime en déformation plane (2D), avec le modèle "Mohr-Coulomb" ou avec le modèle "Soft-Soil", d'estimer la capacité ultime à l'arrachement d'une modélisation (3D) équivalente avec le modèle "Mohr-Coulomb".

Finalement, nous avons trouvé une assez bonne concordance entre les enveloppes de rupture obtenues à partir de l'expression analytique (SUPACHAWAROTE et al., 2004) et celles obtenues des simulations numériques Plaxis ${ }^{\circledR} 3 \mathrm{D}$. Les enveloppes de rupture peuvent donc bien être construites à partir des expressions analytiques, et à partir de l'obtention des charges ultimes verticales $V_{u l t}$ et horizontales $H_{u l t}$ obtenues par simulation numérique.

\section{$8 \quad$ Références bibliographiques}

1 AUBENY C.P., HAN S.W., MURFF J.D. (2003). Inclined load capacity of suction caissons. International Journal for Numerical and Analytical Methods in Geomechanics, Vol. 27, pp 1235-1254.

2 COLLIAT J.L., DENDANI H. (2002). Girassol: Geotechnical design analyses and installation of the suction anchors. Offshore Site Investigation and Geotechnics SUT, Nov., London, UK, pp 107-117.

3 EQUIHUA ANGUIANO L.N., FORAY P. (2007a). Modelling the effects of short- and long-term loading on suction caissons using 2- and 3-finite element methods. $17^{\text {th }}$ Int. Offshore (Ocean) and Polar Engineering Conference, ISOPE 2007, Lisbon, Portugal, July 1-6, pp 1478-1485.

4 EQUIHUA ANGUIANO L.N., FORAY P. (2007b). Modelling of suction caissons using two- and three- dimensional finite element method. International Symposium on Numerical Models in Geomechanics, NUMOG X, Rhodes, Greece, April, pp 425-431.

5 EQUIHUA ANGUIANO L.N. (2008). Modélisation des ancrages de structures offshore flottantes dans les grands fonds marins. Thèse de Doctorat, Institut Polytechnique de Grenoble, $345 \mathrm{p}$.

6 KONDNER, R.L. (1963). Hyperbolic stress-strain response: cohesive soils. J. Soil Mechanics and Foundation Div., ASCE, 89(1), pp 115-143.

7 SUPACHAWAROTE C., RANDOLPH M., GOURVENEC S. (2004). Inclined pull-out capacity of suction caissons. Proc. of the Fourteenth International Offshore and Polar Engineering ISOPE, pp 500-506.

8 THOMAS P.A. (2007). Les différentes plates-formes. La web télévision de l'enseignement supérieur et de la recherche, $U R L$ :

http://www.canal-u.com/canalu/chainev2/utls/programme/27991725 